\title{
Cattle-Related Trauma: A 5 Year Retrospective Review In A Adult Major Trauma Centre
}

John-henry Rhind ( $\nabla$ rhind99@hotmail.com )

Robert Jones and Agnes Hunt Orthopaedic Hospital

Dominic Quinn

Royal Stoke University Hospital

Lucy Cosbey

Royal Stoke University Hospital

\section{Douglas Mobley}

Royal Stoke University Hospital

Ingrid Britton

Royal Stoke University Hospital

Justin Lim

Royal Stoke University Hospital

\section{Research article}

Keywords: Bovine, Trauma, Cow, Bull, Farmer, Animal, Injury

Posted Date: July 22nd, 2020

DOI: https://doi.org/10.21203/rs.3.rs-34195/v1

License: @ (i) This work is licensed under a Creative Commons Attribution 4.0 International License. Read Full License 


\section{Abstract}

\section{Background:}

Bovine injuries are a common and significant cause of trauma, often requiring admission and operative treatment. We review all bovine related injuries over five years, both emergency and GP referrals at an adult major trauma centre in England.

Method:

Retrospective evaluation was undertaken using keywords through radiology referrals and hospital admissions speciality databases. By searching patient notes, demographics were collected as well as the mechanism and the situation of injury; trauma scores were calculated using: Injury Severity Score (ISS) and Probability of Survival (Ps19). Results were divided into emergency patients and GP referrals.

Results:

Sixty-seven patients were identified retrospectively over 5 years, 44 emergency patients (including 23 major traumas) and 23 GP referrals. Combined (Emergency \& GP) mean age 52 years old. 67\% male. Mean ISS 11. Most common combined mechanism of injury, kicked $(n=23)$. In emergency patients, trampling injuries were most common. $86 \%$ of the trampled patients were major traumas and associated with increased ISS (mean 13). Indirect injuries mainly involved farm gates (92\%). $73 \%$ of bull-related injuries were major traumas and had increased ISS scores (mean 17). Orthopaedics was the most common admitting speciality followed by cardiothoracic \& neurosurgery. In emergency patients fractures were the most common primary injury $(n=20)$, upper limb followed by spine. In GP, soft tissue injuries were the most common primary injury. $70 \%$ of the emergency referrals required admission and $50 \%$ operations. Fracture fixation was the most common operative procedure. Only one GP referral required an operation. There were significant delays in GP patients presenting. Two patients had a Ps19 score $<90$. There were two mortalities.

\section{Conclusion:}

Cattle related injuries are a significant cause of severe morbidity and mortality. They are under-reported. Patterns of injury are similar to high velocity road traffic collisions and bull-related injuries or trampling in particular, should alert the clinician to more significant trauma. Farm gates are a frequent cause of trauma associated with cattle. GP referrals with ongoing symptoms for more than two weeks seeking medical advice should alert the clinician to a more serious diagnosis.

\section{Background}

Cattle related injuries are serious, common and often under-reported. On average, there are 4-5 cattle related deaths per year throughout the UK, with around 90 cattle related fatalities since 2000 [1].

The majority of these injuries are associated with farmers and farming communities, up to 24\% [2]. However, non-farming members of the general public are also at risk, particularly in England, where there is a considerable network of footpaths crossing through farms and fields with public access. In addition, the Countryside and Rights of Way Act 2000 (CROW) [3] gave the public the right to walk on mapped access land which includes mountain, moor, heath, down and registered common land. Fifty-four cattle attacks were reported in the UK media to members of the general public, walking, from January 1993 to May 2013 [4]. However, the full extent is unknown.

Patients who present to hospital following cattle related accidents should be treated as high-velocity trauma victims as the pattern of injuries is similar to those seen in road traffic accidents [5]. This is an important issue for rural trauma teams to consider as, in 2018, approximately 309,000 people in England worked on commercial agricultural holdings looking after around 5.3 million cattle and calves [6]

It is difficult to get an accurate figure for non-fatal cattle related trauma as the majority go under-reported or are managed by the General Practitioner (GP) [7]. There have been no previous studies examining the impact of cattle related trauma in the United Kingdom at a major trauma centre in a rural location examining both general practice and emergency admissions. A retrospective 
study was performed over five years to determine the nature and severity of these injuries seen at a rural adult major Trauma centre in England.

\section{Methods}

\section{Data Collection:}

We conducted the study in the Royal Stoke University Hospital, an Adult Major Trauma Centre in the county of Staffordshire, England. It serves a population of 3 million, including North Wales [8].

Only patients directly injured by cattle were included in the results over five years (3/10/2014-3/10/2019). Indirect injuries, such as road traffic accidents, were excluded.

We conducted the search, 3 October 2019. Two independent authors searched our trauma admissions database retrospectively using the following keywords "cattle" "cow" "bull" "bullock" "ox" "oxen" "farm" "farmer". Similarly, the Orthopaedic, Cardiothoracic, Neurosurgery, Maxillofacial, ENT, General Surgery, Urology and Medical admission databases were also searched with the keywords. Individual referrals or admissions matching the keywords were checked to ensure the trauma was bovine related.

Electronic imaging requests stored on the hospital data system were also searched using the same keywords retrospectively. Each matching patients' imaging and records were checked to ensure the trauma was bovine related.

All relevant referral types seen in our hospital within the specified data range were included: Major trauma referrals, Emergency department referrals and GP direct access patients. Major Trauma referrals were defined by the Trauma Audit and Research Network - TARN Criteria - England \& Wales Nov 2019 [9].

Mechanism of injury was ascertained from the clinical notes and classified into broad groups: unknown, kicked, charge/headbutt, body contact, indirect and trampled. Indirect referred to those, for example, injured indirectly between a gate a and a cow. The situation of the injury was recorded where the information was declared in the patient notes; this fell into distinct categories: unknown, herding, slaughtering, vaccinating, milking, manual labour, walking by. The term walking by was used when there was no specific task or event recorded at the time they were injured. Demographics were recorded, and occupation was recorded from the terms the patient used on admission or from the admitting doctor's notes.

Injuries were grouped according to the major primary injury. The injury severity score (ISS) was calculated for all patients from the patient notes. In the major trauma patients, the Probability of Survival was calculated using the Ps19 method, using available data from patient records [9].

\section{Data analysis:}

The ISS was one of the first trauma scores established [10]. Based on the abbreviated injury system (AIS) it produces a single value which correlates with the description of injury and outcome. It is one of the most common trauma scoring systems worldwide, and a score of 16 represents the cut-off for approximate predictive mortality of $10 \%$ [11]. ISS was calculated for all patients. The probability of survival (Ps19) method is an updated value of the TRISS method of probability of survival that incorporates Glasgow coma scale (GCS), comorbidities and outcomes at 30 days [12] [9]. Ps19 was calculated in the major trauma patients using patient records.

Microsoft Excel (Microsoft corp., WA, USA) was used to record data anonymously. ISS and Ps19 scores were calculated by the trauma directorate data analyst team local to our hospital. Prism v. 8.4 (GraphPad, CA, USA) was used for graphs.

\section{Results}

\section{Emergency Referrals}

Forty-four patients were referred from the emergency department within the 5-year data range (3/10/2019-3/10/2014), including 23 major traumas and two mortalities. 
$70 \%$ of the patients were male.

Age (mean 42 years old), majority of patients $>50$ years old $(n=24)($ Fig. 1$)$.

Farming was the most common occupation $(n=20)$. Three others were identified as living on a farm and there were two vets. The occupation was unknown for the remaining patients $(n=19)$.

Eleven of the episodes were bull-related, $73 \%$ of these were major traumas.

Trampling was the most common mechanism in emergency referrals $(n=14) ; 86 \%$ of these were major traumas.

Indirect trauma was the second most mechanism of injury $(n=12)$. Indirect trauma consisted of 11 episodes of trauma sustained whilst leading a cow through a gate and being injured indirectly by the gate (such as being crushed between the gate and the wall or beneath the gate as the cow passed through) and one episode of indirect trauma from the chain a cow was being led on. Kicked was the third most common $(n=11)$. Body contact consisted of events where the cow fell or stumbled on to the patient $(n=2)$

(Fig. 2).

The situation of injury was unknown in $39 \%$ of the patients. In those recorded: walking by (36\%) was the most common situation of injury, within that category three were walking with dogs (Table 1)

Table 1

Situation of Injury of all Emergency patients

\begin{tabular}{|ll|}
\hline Situation of Injury & $\mathbf{N}$. of Patients \\
\hline Unknown & 17 \\
\hline Walking by & 16 \\
\hline Milking & 3 \\
\hline Injection & 2 \\
\hline Manual labouring & 2 \\
\hline Herding & 1 \\
\hline Feeding & 1 \\
\hline Slaughtering & 1 \\
\hline Cleaning hooves & 1 \\
\hline
\end{tabular}

Overall, fractures were the most common primary injury sustained $(n=20)$. Within that category, upper limb fractures were the most common $(n=9)$, spine fractures $(n=5)$ were the next most common type of fracture followed by lower limb $(n=4)$. Blunt chest injury $(n=8)$ was the second most common primary injury followed by soft tissue injuries $(n=7)$. Both long bone fractures were tibial, one was an open fracture requiring multiple washouts and external fixation, the other Intramedullary nailing (Fig. 3).

$70 \%$ required admission into hospital. 50\% underwent an operation.

Orthopaedics was the most common admitting speciality, followed by cardiothoracic and neurosurgery.

Overall, fracture fixation procedures were the most common $(n=12)$. Of these, spinal fixation was the most common type of fracture fixation carried out $(n=5)$ followed by chest wall reconstruction $(n=3)($ Table 2$)$. 
Table 2

Operations of all Emergency patients

\begin{tabular}{|ll|}
\hline Operation & N. of Patients \\
\hline Chest wall reconstruction & 3 \\
\hline Spine Open Reduction Internal Fixation & 5 \\
\hline Humerus Open Reduction Internal Fixation & 1 \\
\hline Finger Exploration Washout & 1 \\
\hline Facial reconstruction & 1 \\
\hline Laparotomy with liver packing & 1 \\
\hline Laceration wash and closure & 2 \\
\hline Forearm Open Reduction Internal Fixation & 1 \\
\hline Nailbed repair & 1 \\
\hline Intramedullary Nail Tibia & 1 \\
\hline External fixation Tibia open fracture & 1 \\
\hline Endarterectomy of femoral artery plus thrombectomy & 1 \\
\hline Ankle Open Reduction Internal Fixation & 1 \\
\hline Wrist Open Reduction Internal Fixation & 1 \\
\hline Washout closure scalp laceration & 1 \\
\hline
\end{tabular}

There were 23 major trauma patients, within the emergency referrals, as defined by the TARN criteria for major trauma [9].

All the major traumas were admitted, 70\% underwent operations.

$52 \%$ of these patients had an ISS $>16$.

Two patients had a Ps19 score $<90 \%$.

There were two mortalities $(n=2)$ within the major trauma referrals.

$91 \%$ of the major trauma patients had a complete data set (physiological parameters, observations charts, comorbidities and 30 day outcomes) to calculate Ps19 (Fig. 4).

\section{GP referrals}

There were 23 GP Direct Access patients referred within the date range.

$70 \%$ were male.

Mean age 53 years old (Fig. 1).

The situation of injury was unknown in GP patients due to less detail in patient records.

The most common mechanism was kicked $(n=12)$.

The majority of GP referrals were soft tissue injuries $(n=16)$. There were three rotator cuff injuries with delayed presentations (Fig. 3). None of the patients required admission; however, one of the hand fractures was a scaphoid fracture requiring operative fixation at a later date after a failed trial of conservative management due to delayed presentation. 
The GP referrals had a varied time to presentation ranging from 2 days to 3 months, mean ( $n=25$ days).

Three patients have an ISS > 5 (Fig. 5).

\section{Combined Results (Emergency and GP)}

Sixty seven patients overall.

Combined mean age 52 years old (Fig. 1).

Most common mechanism kicked $(n=23)$ (Fig. 2).

Overall, the most common primary injury was soft tissue injury $(n=23)$ followed by fractures $(n=22)$ (Fig. 3$)$. $27 \%$ of patients had an ISS $>16$ (Fig. 5). The overall mean ISS was 11.

\section{Discussion}

In this retrospective study, 67 patients were identified over 5 years. Double the incidence reported in a similiar retrospective study by Murphy et al in Ireland, where 47 patients were identified over 10 years [13]. This may be due to more comprehensive retrospective search techniques using radiology search records in addition to searching patient databases. It may also reflect how under reported these injuries are, particularly in the British Isles. However, in another prospective study in New Zealand the incidence was higher [14].

Male working farmers more than fifty years old were the most common demographic in our study. Not only was this demographic more common, but their injuries were more severe and consisted of more polytraumas, supporting other literature [15] [13] [16]. Patterns of injury in bovine-related trauma are described as similar to road traffic collisions [5], whereas road traffic collisions typically occur in young male patients bovine trauma is more frequent in older patients.

Walking by was the most common situation of injury in emergency patients. In three of these situations, they had dogs, which are a recognised risk factor for trauma with cows [4]. Milking was the next most common situation in this study. Dairy farming is common in the local county, Staffordshire, making this unsurprising and is a risk [17]. There were two injuries from injections whilst vaccinating, sustained by vets; Lucas et al. recognised this risk with veterinarians [18]. Slaughtering was another situation identified; tagging new-born calves and clipping cattle before slaughter has been identified as a risk factor for injury in previous literature [2]. 39\% of the situations in the emergency patients and all the situations in GP patients were unknown, highlighting the limits of this retrospective study.

Another established risk factor is maternal behaviour in cows with calves [19]; no calves were identified in this study. It is unclear if this was due to the limitations of this study, or there were none present.

In terms of mechanism, kicking was the most common when all emergency and GP referrals were combined. However, trampling was more common in emergency referrals and associated with more major traumas (86\%) and higher ISS scores - mean 13 (overall mean 11).

Indirect injuries were the second most common mechanism in emergency referrals (third overall), $92 \%$ of these involved moving cattle through farm gates and crush injuries. $58 \%$ of these episodes were major traumas and were associated with higher ISS scores - mean 14 (overall mean 11). It is unclear if current gates being used are inadequate or are incorrectly used, in which case, further education is required. The Health and Safety Executive (HSE) offers safety advice on cattle for farmers and landowners and recommends that gates are regularly checked and maintained [20]. There is no mention of what constitutes an adequate safe gate. Watts et al. also found being crushed by the gate, a common mechanism [14]. Browning et al. identified that limiting the size of cattle herds reduced injury, mainly through gates, as well as the use of all-terrain vehicles (ATV) [21]. Introducing minimum safety standards for gates and maximal herd sizes in the UK may be a useful addition.

Bull injuries, although less frequent, were also associated with more major traumas (73\%) and higher ISS scores - mean 17 (overall mean 13). One of the two mortalities was bull related [16] [22] [15]. 
Overall, blunt over penetrative trauma was more common and associated with more severe trauma in this study, supporting the literature [23] [13].

The most common primary injury in emergency patients was fractures, supporting other literature [13] [15]. Overall, upper limb fractures were the most common category of fracture, similar to other studies[18] [14], followed by the spine. $80 \%$ of the spine fractures were in male farmers more than seventy years old, and all involved thoracic fractures. This may highlight an additional injury pattern seen in elderly male working farmers. When the primary injury was combined with GP patients, the most common injury was soft tissue injuries.

Elderly male farmers sustaining blunt head and chest injuries have been identified as the most at-risk group for mortalities associated with cattle [24]. Both mortalities in this study were male farmers over fifty years old, sustaining blunt trauma, one a head injury while the other suffered multiple abdominal injuries and spinal fractures. This demographic may need to consider extra precautions such as body armour or alternative working practices.

The GP patients had significantly delayed presentations. It is likely that there are even more injuries that go unrecorded as patients, particularly with soft tissue injuries, will have an improvement in their symptoms before presenting and no longer seek medical advice. This is particularly prevalent in the farming community who are often self-employed.

Although most of the GP injuries were soft tissue, there were some important diagnoses initially missed such as scaphoid fractures and rotator cuff tears. This highlights the importance of investigating these injuries fully in patients with ongoing symptoms that have not improved over a significant amount of time. There are no previous studies we could find that have have been set in a major trauma centre and included general practice injuries, in the United Kingdom. Doyle et al. described farmingrelated injuries in general practice in Ireland [7].

The most common operative procedures were upper limb fracture fixation and spine fracture stabilisation; all these patients had unstable thoracic spine fractures or combined thoracolumbar, thoracic-cervical injuries. Chest wall reconstruction was the third most common operation type. The majority of these operations were associated with blunt trauma.

Orthopaedics was the most common admitting speciality confirming that fractures were the most common overall injury associated with bovine trauma. This was followed by Cardiothoracic and neurosurgery. This highlights the significant chest and head injuries found in this study which are most associated with mortality [24].

Out of all animal-related injuries, cattle injuries cause the most hospital admissions [22] [25]. Additionally, previous studies have indicated bovine injuries are more expensive to treat than other animal-related injuries [26]. We were unable to calculate the cost of each admission in this study. Watts et al. calculated the total hospital cost for 78 patients in New Zealand with bovine-related injuries as $\$ 32,884$ and total loss of income as $\$ 86,178$ [14].

The chief limiting factor in this study is its retrospective design. The data systems in the hospital rely on user input to collect information such as the mechanism of the injury, which may not always be accurate. There is also no specific coding system for bovine-only related injuries in either the ICD 9 or ICD 10 classification manuals if the patient is admitted to the hospital. Additionally, many people do not seek primary or secondary care when they are injured. Only the injuries in which the doctors document the causative animal were picked up through our methods. Some of the identified patient's notes were also incomplete or lacked key details to allow more detailed analysis of injury patterns or calculation of trauma scores.

Introducing specific hospital coding (ICD classification) for bovine injuries would allow further research in this area to be carried out more easily.

Future research would also benefit from more prospective level one and two studies dedicated to collecting all the available patient information and negating the limits of this study. We identified only one prospective study, carried out in New Zealand [14].

In Sweden, animal registries are kept in some rural communities to monitor the nature of these animal injuries, which may be a useful addition in this setting [26]. 


\section{Conclusion}

Cattle related injuries are a significant cause of severe morbidity and mortality. They are under-reported and more awareness should be raised, as a public health risk. Patterns of injury are similar to high velocity road traffic collisions and Bull-related injuries or trampling in particular, should alert the clinician to more significant trauma. Farm gates are a frequent cause of trauma associated with cattle. GP referrals with ongoing symptoms for more than two weeks seeking medical advice should alert the clinician to a more serious diagnosis. Clinicians should maintain a high suspicion for upper limb and spinal fractures. Further prospective research is required on this important topic.

\section{Abbreviations}

GP General Practice

ISS Injury Severity Score

Ps19 Probability Survival Score

\section{Declarations}

Ethics approval - a local ethics committee ruled that no formal ethics approval was required

All authors Consent for publication

Availability of data and material - happy to share excel files if required

No Competing interests

Funding - not applicable

Authors' contributions - all authors read and edited the manuscript, LC and IB provided radiology expertise in gathering relevant imaging and data and processing data, DM provided major trauma data, DQ helped process data and with results, JL reviewed and edited whole manuscript. All authors have read and approved the manuscript.

Author declaration: no conflict of interest

Acknowledgements - not applicable

Consent for publication - all authors agree to consent to publication

\section{References}


[1] HSE, "Summary of fatal injuries in agriculture, forestry and fishing in Great Britain 2018/19," 2019. [Online]. Available: http://www.hse.gov.uk/agriculture/pdf/agriculture-fatal-injuries-1819-summary.pdf.

[2] S. Lindsay, S. Selvarag, J. Macdonald and D. Godden, "Injuries to Scottish farmers while tagging and clipping cattle: a cross-sectional survey," Occupational Medicine, vol. 54, no. 2, 2004.

[3] U. P. G. Acts, "legislation.gov.uk," 2000. [Online]. Available: http://www.legislation.gov.uk/ukpga/2000/37/contents.

[4] F. Angharad, M. Mcintyre and C. Westgarth, "Are cattle dangerous to walkers? A scoping review," BMJ Journals, vol. 22, no. 6, 2015.

[5] A. Nogalski, L. Jankiewicz and G. Cwik, "Animal related injuries treated at the Department of Trauma and Emergency Medicine, Medical University of Lublin.," AAEM, 2007.

[6] DEFRA, "Farming statistics - final land use, livestock populations and agricultural workforce as at 1 June 2018 ," 2018. [Online]. Available: https://assets.publishing.service.gov.uk/government/uploads/system/uploads/attachment_data/file/805472/structurejun2018final-eng-31may19.pdf.

[7] Y. Doyle and R. Conroy, "The spectrum of farming accidents seen in Irish general practice: a one-year survey," Family Practice, 1989.

[8] "UNHM University Hospital North Midlands," 2020. [Online]. Available: http://www.uhnm.nhs.uk/news/pages/UHNMplays-part-in-Major-Trauma-success.aspx.

[9] T. A. R. N. (TARN), "The Trauma Audit and Research Network - Procedures Manual England \& Wales," Nov 2019. [Online]. Available: https://www.tarn.ac.uk/Content.aspx?ca=4.

[10] B. SP, O. B, H. W and L. W, "The injury severity score: a method for describing patients with multiple injuries and evaluating emergency care," J Trauma, 1974.

[11] C. HR, C. WS, S. WJ, L. MM, K. SL and B. JR, "The Major Trauma Outcome Study: Establishing National Norms for Trauma Care," J Trauma, 1990.

[12] C. Boyd, M. Tolson and C. WS, "Evaluating trauma care: the TRISS method. Trauma Score and the Injury Severity Score.," J Trauma, 1987.

[13] c. murphy, c. mcguire, n. o'malley and p. harrington, "Cow-related trauma: A 10-year review of injuries admitted to a single institution," Injury, pp. 548-50, 2010.

[14] M. Watts and E. Eisel, "Cattle associated trauma - A one year prospective study of all injuries," injury, vol. 42, no. 10, pp. 1084-87, 2011.

[15] s. shriyan, u. mani, f. bhot, e. sada, r. ursekar and d. adake, "Animal Injuries: a Case Series of Bull Induced Injuries in India," adv j emerg med, 2020.

[16] K. Dogan, S. Demirci, Z. Erkol, G. Sunam and T. Kucukkartallar, "Injuries and deaths occurring as a result of bull attack," $J$ Agromedicine, pp. 191-6, 2008.

[17] S. G. Boyle and R. Gerberich, "Injury from Dairy Cattle Activities," Epidemiology, vol. 8, pp. 37-41, 1997.

[18] M. Lucas, L. Day and L. Fritschi, "Serious injuries to Australian veterinarians working with cattle," Australian Veterinary Journal, vol. 91, 2012.

[19] S. Turner and L. AB, "Relationship between maternal defensive aggression, fear of handling and other maternal care traits in beef cows.," Livestock Scientist, 2007.

[20] H. a. S. Executive, "Cattle and public access in England and Wales Advice for farmers, landowners and other livestock keepers," [Online]. Available: https://www.hse.gov.uk/pubns/ais17ew.pdf.

[21] S. Browning, W. Westneat, S. T and B. Reed, "Cattle-related injuries and farm management practices on Kentucky beef cattle farms," Journal of Agricultural Safety and Health, vol. 19, no. 1, pp. 37-49, 2013.

[22] H. Busch, T. Cogbill, L. J and L. BO, "Blunt bovine and equine trauma," J Trauma, pp. 559-60, 1986.

[23] h. shukla, d. mittal and y. naithani, "Bull horn injury: a clinical study," injury, pp. 164-67, 1977.

[24] C. f. D. C. a. P. (CDC), "Fatalities caused by cattle-four States, 2003-2008," MMWR Morb Mortal Wkly Rep, vol. 58, no. 29, pp. 800-4, 2009.

[25] C. L, "The maul of the wild. Animal attacks can produce significant trauma," Emergency Medicine Services, 1994.

[26] U. Bjornstig, A. Eriksson and A. Ornehult, "Injuries caused by animals," Journal of Injury, pp. 295-8, 1991.

Page 9/13 
Figures
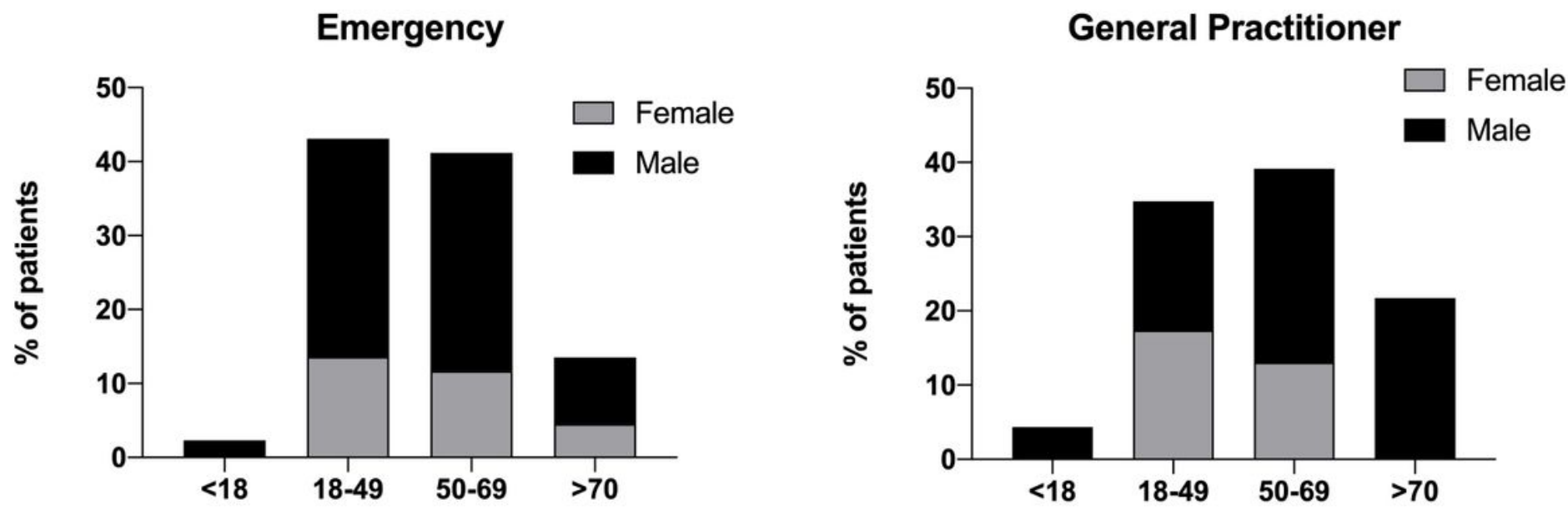

Figure 1

Age Range of all patients

Mechanism of Injury

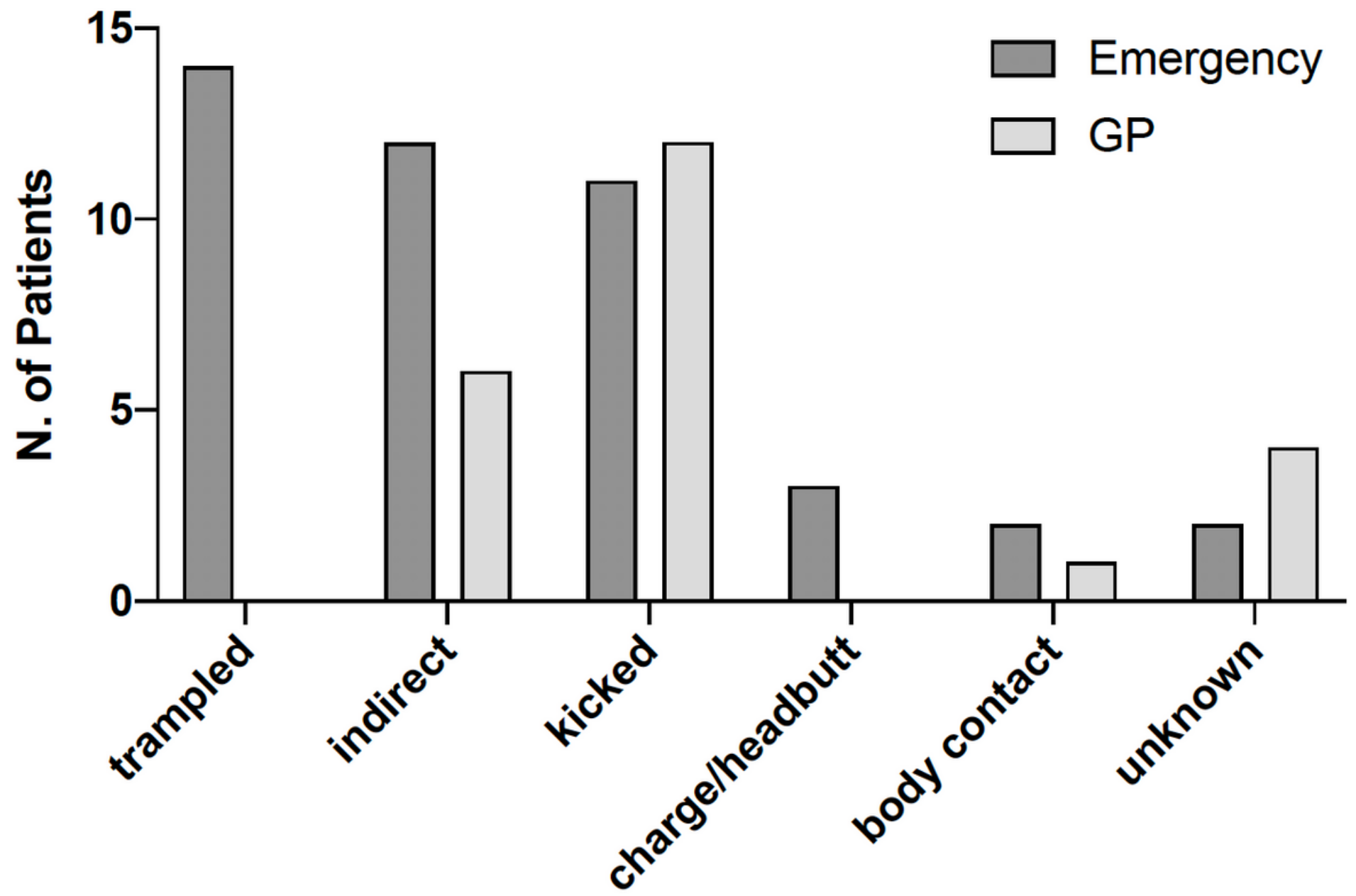


Figure 2

Mechanism of Injury of all patients, GP: general practitioner

\section{Primary injury sustained}

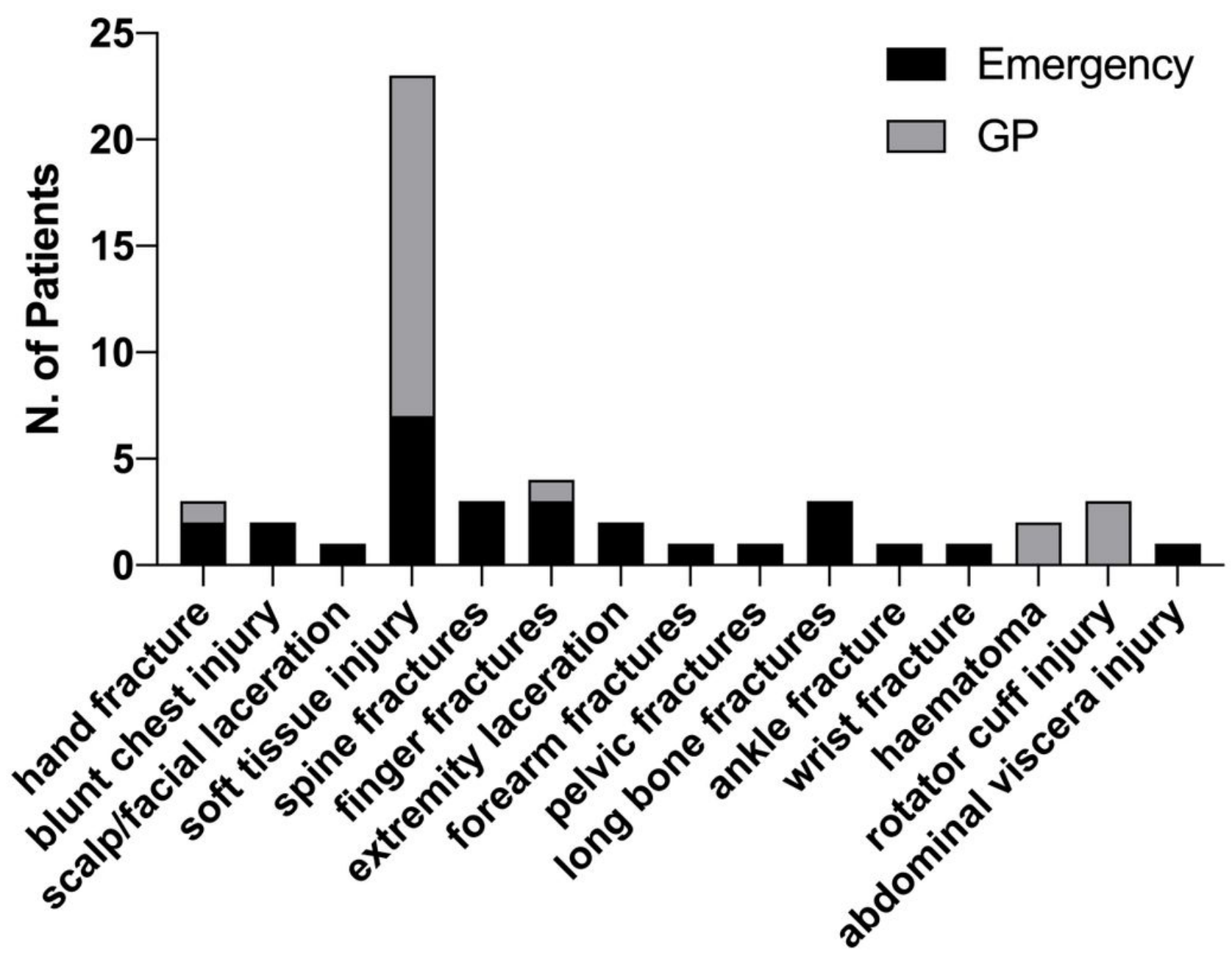

Figure 3

Primary injury sites of all patients, GP: general practitioner 


\section{Probability Survival}

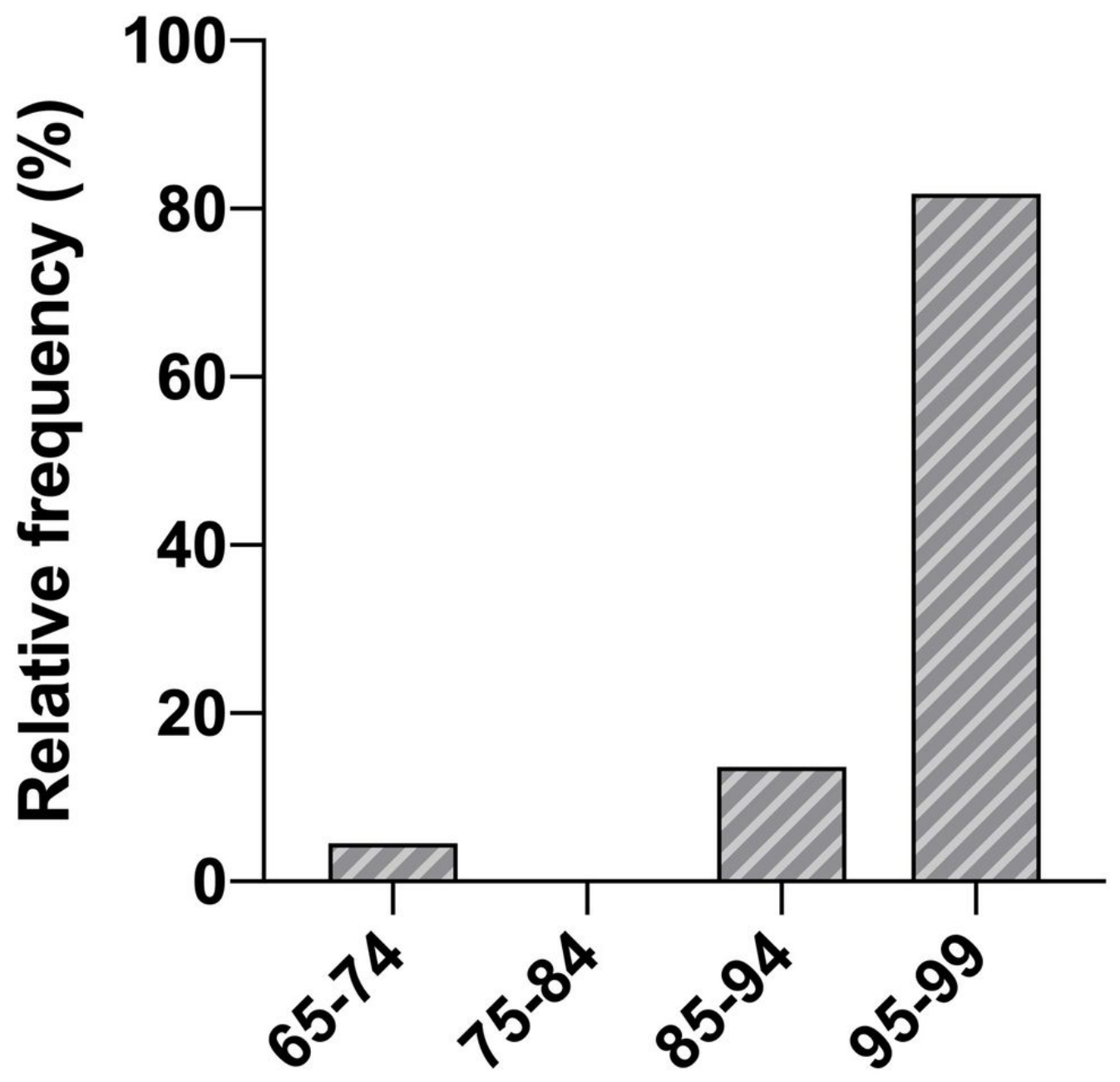

Figure 4

probability of survival (Ps19) for major trauma patients 


\section{ISS}

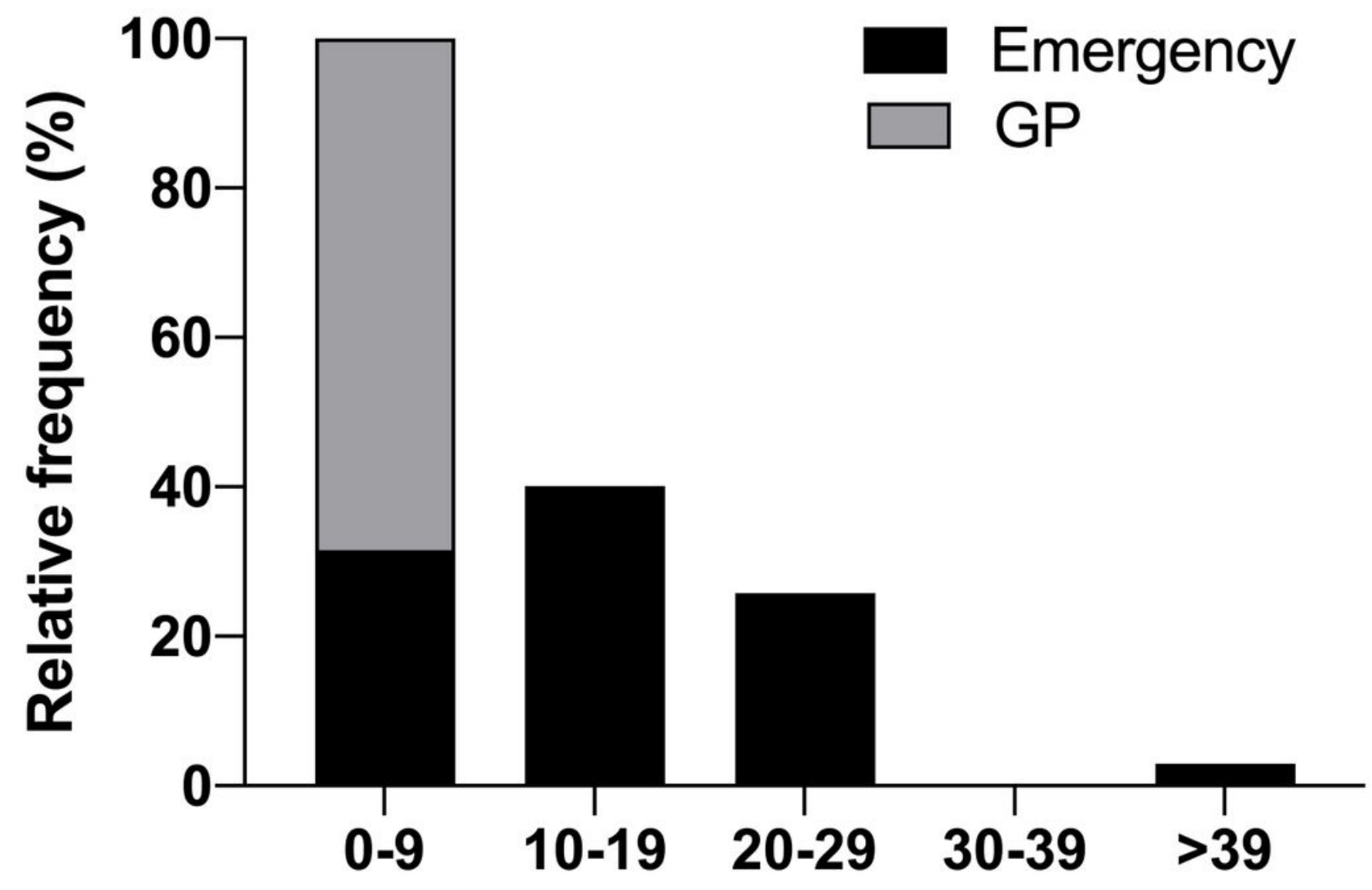

Figure 5

Injury Severity Score (ISS), GP: general practitioner 